Article

\title{
Performance Improvement of Ethernet-Based Fronthaul Bridged Networks in 5G Cloud Radio Access Networks
}

\author{
Muhammad Waqar(D and Ajung Kim * \\ Department of Optical Engineering, Sejong University, Seoul 143747, Korea \\ * Correspondence: akim@sejong.ac.kr; Tel.: +82-10-4597-7542
}

Received: 9 June 2019; Accepted: 12 July 2019; Published: 15 July 2019

Featured Application: The results of the research can be used in the practical deployments of the leveraging Ethernet fronthaul bridged networks in the 5G cloud radio access networks.

\begin{abstract}
Cloud radio access networks (C-RANs) are emerging architectural solutions to anticipate the increased capacity and quality demands of future $5 \mathrm{G}$ cellular networks at a reduced cost. In C-RANs, a transport segment referred to as fronthaul has been defined, which become a major constraint in practical implementations due to its high cost. A transport protocol referred to as eCPRI (enhanced common public radio interface), which was specifically designed for the fronthaul networks, imposes stringent end-to-end (E2E) latency and capacity requirements, which can be satisfied through the extortionate optical links. The high implementation cost of optical fronthaul networks significantly increased the system cost and made the fronthaul a hurdle to accomplish the cost-benefits of the C-RANs' architecture. The globally deployed Ethernet networks could be leveraging solutions, but are inadequate to comply with the eCPRI requirements in fronthaul bridged networks and result in intolerable latencies due to ineffectual traditional quality of service aware forwarding schemes. Therefore, to realize the cost-benefits of ubiquitously deployed Ethernet infrastructure, this paper proposes the E2E latency aware path computation and packet forwarding schemes, which ameliorate the performance of Ethernet-based fronthaul bridged networks to transport the eCPRI traffic at tolerable latencies. The simulation results verify the feasibility of low-cost Ethernet to carry the eCPRI traffic streams up to $100 \mathrm{Gbps}$ with the proposed schemes in fronthaul bridged networks.
\end{abstract}

Keywords: 5G networks; BBU centralization; cloud radio access networks; eCPRI; fronthaul

\section{Introduction}

According to a recent prediction [1], the mobile data traffic will increase 10 times from 2017 to 2022, and more than 50 billion devices will be connected to the existing infrastructure by 2020. A transition from the static radio access networks (RANs) to flexible and reconfigurable networks through the virtualizations and cloudification was highly desirable to anticipate the traffic demands of future 5G cellular networks. Recently, cloud radio access networks (C-RANs) have been proposed, which are enhancements of classical RAN architectures through the cloudification and virtualizations techniques to comply with the requirements of envisioned 5G mobile networks. In C-RANs, the base station functionalities are split into distributed units known as the remote radio heads (RRHs) and central units known as the baseband units (BBUs), which are virtualized and pool at a central cloud [2]. The C-RANs approach brings several advantages for the network operators, including a reduction in the operational, management, and energy costs, as well as effective implementations of the coordinated multipoint (CoMP) transmission and reception schemes for the inter-cell interference mitigations [3]. With the 
virtualization of base station functionalities, the scalability of RAN architectures has been improved, and the spectral efficiency of the operator's network can be increased. Furthermore, a number of RRHs can be installed at the cell sites by simply connecting with the BBU pool, which reduces the cost of deploying expensive baseband processing units nearer to the RRHs at the cell sites [4].

The 3rd generation partnership project (3GPP) [5] defined the eight functional splits for the C-RANs in order to connect the RRHs and the BBU pool. A chosen functional split uniquely defines the properties of the system design such as the complexity, costs, latency, data rates, throughputs, and achievable statistical multiplexing gains. In a low-level functional split, which has the maximum benefits of performance and cost efficiency, all the higher layer functions are moved into the BBU pool, and only the radio frequency (RF) functions are employed in the RRHs at a cell site [6]. This requires a high-speed communication link between the RRHs and the BBU pool, which is referred to as the mobile fronthaul (MFH). The fronthaul segment is connected to the core network through the backhaul links [7]. Figure 1 depicts the considered MFH networks of the C-RANs architecture.

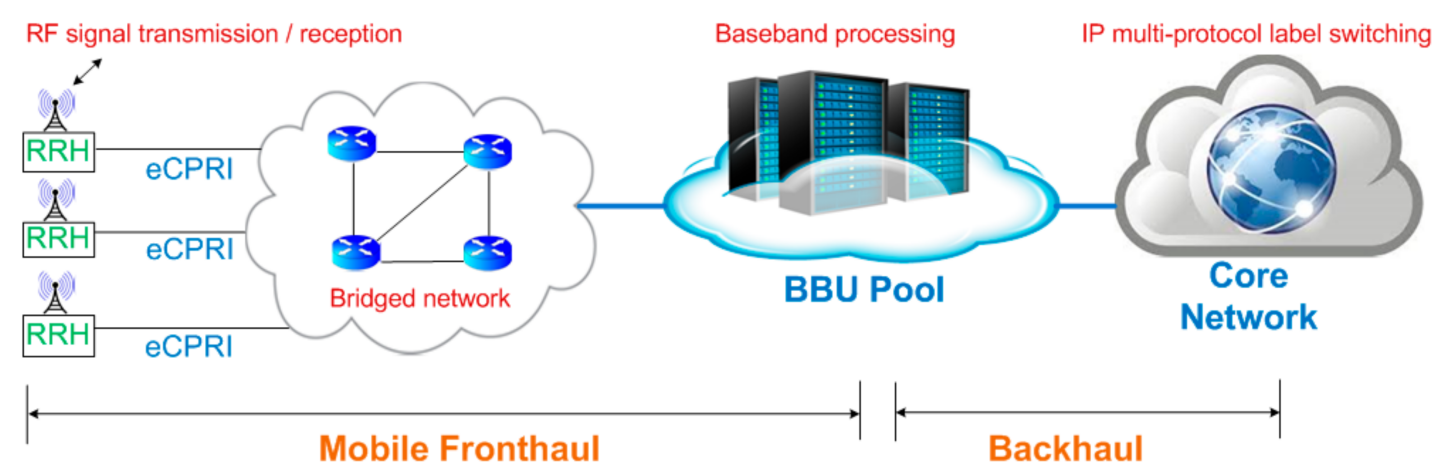

Figure 1. Cloud radio access networks (C-RANs) architecture for envisioned 5G cellular systems.

A MFH carries the digitized in-phase and quadrature (IQ) samples of baseband signals through the common public radio interface (CPRI) [8]. The CPRI is a non-packetized protocol that was specifically designed for the fronthaul networks by the leading telecom vendors, and cannot be integrated with other packetized transmissions unless a circuit (e.g., a wavelength) is reserved for it [9]. In this work, we consider a recent evolution of CPRI that is eCPRI (enhanced-CPRI) [10], which can packetize the IQ samples within Ethernet frames and is fully compatible with Ethernet systems. The eCPRI-based MFH networks are expected to deliver the throughputs of $10 \mathrm{Gbps}$, packet delay variations (PDVs) within $100 \mathrm{~ns}$, and end-to-end (E2E) latency of less than $250 \mu$ s for all the RRHs streams [11,12]. The aforementioned requirements can only be fulfilled with the expensive and extremely high-capacity optical links. However, the cost of deploying such optical fronthaul bridged networks significantly increased the capital expenditure (CAPEX) and operational expenses (OPEX) for the network operators. As a result, the cost benefits of the C-RANs architecture could not be realized [13]. Thus, the implementation of low-cost MFH becomes a challenging issue in the C-RANs [14].

The 10 gigabit Ethernet systems can be more economical and remunerative solutions as compared to the optical networks to carry the eCPRI traffic in the MFH. Moreover, Ethernet supports the virtualization techniques and OAM (operations, administration, and management) functionalities, which make them more suitable to connect the RRHs with the BBU pool through the fronthaul bridged networks. However, the Ethernet networks that use the legacy quality of service (QoS)-aware routing [15] and packet forwarding schemes [16] yield the per-hop latency of about one millisecond and PDVs of up to hundreds of microseconds, which are much higher than the eCPRI traffic requirements $[10,17]$. A major cause in Ethernet systems for not complying with the eCPRI requirements is the non-consideration of frame-level queuing delays at the bridging nodes, which are caused by the globally synchronized eCPRI bursts flowing to and from the RRHs at different line rates. Despite the utilization of the 
QoS-aware forwarding schemes in the optical fronthaul bridged networks, the queuing delays among the burst of eCPRI streams are high, which increases the E2E latencies to an unacceptable level [18]. The queuing delays and E2E latencies become more critical in capacity-constraint Ethernet-based fronthaul bridged networks (EFBNs) [13]. For enhancing the capability of economically affordable and ubiquitously deployed Ethernet networks to transport the eCPRI streams at tolerable latencies, a novel packet forwarding mechanism that would efficiently utilize the limited Ethernet bandwidths and yield the low queuing delays at intermediate bridges is vital. Therefore, this paper proposes the E2E latency-aware path selection and packet transmission schemes that guarantee the low queuing delays in the EFBNs and retain the E2E latencies of maximum eCPRI streams closer to the threshold by taking advantage of the virtualization and OAM capabilities of the Ethernet systems. The proposed latency-aware path selection scheme assures the lowest E2E latencies in the fronthaul bridged networks by considering the frame-level queuing delays of eCPRI bursts. The proposed packet forwarding scheme mitigates the delays of eCPRI streams that experience high latency by slightly increasing the queuing delays of traffic streams that experience low latency. As a result, for all traffic streams, tolerable E2E latencies can be realized in the EFBNs. The computer simulations are undertaken on varied and realistic scenarios. The simulation results confirmed that the proposed schemes maximize the simultaneous transmissions of eCPRI streams of up to $100 \mathrm{Gbps}$ without violating the QoS requirements as well as improve the link distances, whereas such performance is not guaranteed with the existing schemes. Moreover, the transmissions of time-sensitive eCPRI streams at tolerable latencies with the proposed schemes through the leveraging Ethernet links significantly reduced the CAPEX and OPEX of deploying extortionate optical links in fronthaul bridged networks. Hence, without hardware upgradations, the performance of low-cost Ethernet networks can be improved with the proposed schemes, which can be programmed at the intermediate nodes of the EFBNs using the OpenFlow protocols and software-defined networking (SDN) techniques [19].

The rest of the manuscript has the following organization. Section 2 introduces the state-of-the-art work on the CPRI and Ethernet-based fronthaul networks. Section 3 formulates the measurement procedures for the end-to-end latencies and distances in the EFBNs. Section 4 introduces the proposed transport schemes for the EFBNs. Section 5 presents the simulation setup for evaluating the proposed schemes. The results of the proposed schemes and comparison with the existing techniques are presented in Section 6. Finally, conclusions are provided in Section 7.

\section{State of the Art-Ethernet Based Fronthaul Networks}

Due to prior investments and backward compatibility issues, the existing standard bodies are focusing on the Ethernet-based fronthaul implementations. Currently, the IEEE 1914.3 [20] working group is investigating the techniques to encapsulate the CPRI samples within the Ethernet frames. This standard is also working on defining the techniques to improve the statistical multiplexing gains in the CPRI over Ethernet networks. The IEEE 802.1CM [21] is striving to standardize the Ethernet-based fronthaul systems for transporting the eCPRI streams in bridged networks. This standard is in the process of defining the default configurations, procedures, and profiles to carry the time-sensitive eCPRI streams in the MFH at low latencies. However, no mechanism has been defined explicitly in the standards to compensate the constraints of queuing delays at bridges and simultaneously transport the multiple eCPRI streams in Ethernet-based fronthaul bridged networks by satisfying the latency requirements.

Recently, due to the cost-benefits of Ethernet networks over the eCPRI-based optical switching networks, several academicals research proposals have been submitted to carry the time-sensitive fronthaul traffic in Ethernet networks. Most of the initial studies addressed the delay, jitter, and throughput challenges of the fronthaul networks by implementing the priority schemes [22] and packets scheduling [23] protocols, which were initially proposed for time-sensitive networking (TSN). The study [24] implemented the frames priority mechanism and traffic scheduling algorithm for the Ethernet-based MFH networks. This study shows that traffic priority can be effective in minimizing 
the delays in the Ethernet networks, but cannot fulfill the CPRI demands. The author claims that the queuing delays could be mitigated at switches with the traffic scheduling algorithm for a few CPRI streams. However, the parameters and configurations under which the results were obtained were not detailed in the paper. A performance analysis of passive optical networks (PON)-based fronthaul systems for long-range transmissions has been reported in [25]. This study shows that PON systems could not fully satisfy the eCPRI requirements without several hardware upgrades. This study also proposed to employ an expensive erbium-doped fiber amplifier (EDFA) at selected locations of the networks for achieving the QoS parameters within the eCPRI requirements. It is suggested to use an EDFA before the BBU pool for the considered fronthaul scenario to comply with the eCPRI demands. The studies [26] and [27] discussed the traffic scheduling and buffering techniques for Ethernet switching systems to improve the multiplexing gains in the fronthaul networks while retaining the delays and jitter within the CPRI limits. These studies showed that the CPRI traffic flows can be transported through at maximum one or two Ethernet switching nodes without violating the CPRI requirements. However, these schemes become ineffective for retaining the E2E latencies within the limits in more realistic fronthaul bridged networks, which comprises several intermediate bridging nodes between the RRHs and the BBU pool.

The constraint-based and QoS aware routing schemes would be alternative solutions to manage the latencies in multi-hop Ethernet networks. Since then, these routing schemes have been well studied in the past few years to tackle the network constraints such as the cost, capacity, throughputs, and delays. Dijkstra's shortest path algorithm that allocates the weights to different links, based on the available bandwidths and distances, has been studied in [28] for TSN networks, which could be an efficient solution for the delay constraint routing problems. Mutual constraint-based routing procedures have been discussed in [29] to fairly utilize the limited resources in the Ethernet bridged networks. In a study [30], multipath routing schemes were expedited for performing the dynamical load balancing and improving the congestions in TSNs by monitoring and maintaining the various performance indicating parameters. However, the existing QoS-aware routing schemes focus on searching the feasible routes subject to single or multiple QoS constraints, and select the best routes with no consideration of the frame level queuing delays, which is the case in the fronthaul bridged networks due to the simultaneous transmissions of the globally synchronized eCPRI bursts. Moreover, a high control messages overhead with the legacy routing schemes [31] due to the dynamic selection of the paths and continuous monitoring of the networking parameters further increases the delays, which makes them unsuitable for the E2E latency constraints of the EFBNs.

The study [18] proposes a queuing model to improve the delays in optical fronthaul bridged networks. In this study, Nakayama et al. proposed a low latency routing (LLR) mechanism based on the Markov chain Monte Carlo (MCMC) method, which searches the paths for individual streams by considering the traffic load on the different links. This study showed that the legacy QoS-aware routing schemes such as the constraint-based shortest path bridging (SPB) [32] become inefficient to satisfy the latency requirements in the MFH bridged networks, and schemes such as the LLR are required to satisfy the MFH requirements. However, the LLR scheme also yields intolerable E2E latencies, and becomes ineffective at complying with the eCPRI requirements once the traffic load increases to a certain level. This is because the LLR scheme uses a single path for transporting the time-sensitive flows, which are usually the shortest neighboring paths. Under the high load conditions, these alternative paths also get congested and cause intolerable E2E latencies for the eCPRI flows. Moreover, the LLR scheme lacks the mechanism to minimize the queuing delays of eCPRI streams of equal priority at the bridging nodes, which is highly desirable for alleviating the E2E latencies and successfully deploying the leveraging Ethernet-based fronthaul bridged networks in 5G cloud-RANs.

\section{E2E Latency and Distance Formulation}

The MFH networks require high multiplexing gains to efficiently implement the coordinated multiple point schemes in the C-RANs, which can be improved by increasing the aggregation of 
RRHs streams over the fronthaul links. However, the simultaneous transmissions of multiple eCPRI streams from the RRHs increased the E2E latencies to an unacceptable level, especially in the Ethernet networks [24-26]. Currently, the eCPRI-based Ethernet networks are gaining the attention of network operators and telecom vendors due to their low-cost and reconfigurability features, but achieving the required E2E latency is still a challenging issue [27] that requires further exploration. The E2E latency in such networks is the result of indeterministic and deterministic delays. The indeterministic delays such as the encapsulation delay $D_{E n} l_{E n c}$, the queuing delay $D e l_{Q u e}$, and the processing delay $D e l_{\text {Proc }}$ vary non-predictably in the EFBNs, while the deterministic delays such as the propagation delay $D e l_{\text {Prop }}$ and the transmission delay $D e l_{\text {Tran }}$ are pre-determined.

The indeterministic delays such as the $D e l_{E n c p}$ correspond to the mapping delay of the eCPRI IQ samples within the Ethernet payload at the bridging nodes before transmitting them over the outgoing links. In this study, the $\operatorname{Del}_{E n c p}$ for 10-gigabit Ethernet networks is estimated between the $19 \mu$ s to $2 \mu \mathrm{s}$ for mapping the eCPRI flows from line rate option 1 to 7 [10], respectively within the Ethernet payload size of 1500 bytes [26]. However, the $D e l_{E n c p}$ only increases when the traffic flows first enter the Ethernet networks. Therefore, the $D e l_{E n c p}$ values are only considered at the first level of the bridging nodes within which the RRHs are physically connected.

The $D e l_{Q u e}$ is the length of time for which the packets of different streams wait in the buffers of the bridges before transmissions. When the $f$-th flow competes the $g$-th flow for simultaneous transmission over the $n$-th link, these flows are referred to as competitive flows [18]. Hence, the worst-case $D e l_{Q u e}$ experienced by the $f$-th flow over the $n$-th link is calculated from Equation (1):

$$
D e l_{Q u e}=\frac{m \lambda}{C}
$$

where $m$ is the maximum burst size of a flow, and $C$ is the link capacity, while $\lambda$ is the number of competitive flows [18].

The $D e l_{\text {Proc }}$ is the time that the switches take to decode the header of a packet to output them on the egress ports. The $D e l_{\text {Proc }}$ value, including the OpenFlow-based forwarding table lookup delays per bridging node experienced by the eCPRI streams, is estimated as $1.5 \mu \mathrm{s}$ for the considered networks [27]. Thus, the indeterministic delays Del $_{\text {indet }}$ in the EFBNs along a route can be calculated from Equation (2), and the deterministic delays $D e l_{\text {det }}$ can be calculated from Equation (3). Here, the deterministic delays such as $D e l_{\text {Prop }}$ are a measure of the time required for a frame to propagate from one node to the other. The Del $l_{\text {Prop }}$ is equal to $d / s$, where $d$ is the link distance in $\mathrm{km}$, and $s$ is the signal propagation speed in copper (i.e., $2 \times 10^{8} \mathrm{~ms}^{-1}$ ). The $D e l_{\text {Tran }}$ is the time taken by the physical layer at the source to transmit the packets over the link. In this study, the $D e l_{\text {Tran }}$ is estimated as $1.2 \mu$ s based on $L / C$, where $L$ is the data packet length (i.e., 1500 bytes), and $C$ is the channel data rate (i.e., $10 \mathrm{Gbps}$ ). Finally, the E2E latency of the $n$-th eCPRI traffic stream that is carried by a fronthaul link between the RRH and the BBU pool is formulated using Equation (4):

$$
\begin{gathered}
D e l_{\text {indet }}=D e l_{\text {Encp }}+\left[D e l_{\text {Que }}+D e l_{\text {Proc }}\right] \times \operatorname{Link}_{n} \\
D e l_{\text {det }}=D e l_{\text {Prop }}+D e l_{\text {Tran }} \\
E 2 E_{\text {latency }}=D e l_{\text {indet }}+D e l_{\text {det }}
\end{gathered}
$$

Along with the latency, the distance of the fronthaul links between the RRHs and the BBU pool is another important performance indicating parameter for the EFBNs [24-26]. The E2E latency limits the maximum distances in the EFBNs. For commercially deploying the cloud-RANs, improvement in the link distances between the RRHs and the BBU pool is essential. The distance $(\mathrm{km})$ of a fronthaul link in the considered scenario is calculated using Equation (5):

$$
\text { Distance }=\frac{\left(\text { Max threshold }-W E 2 E_{\text {latency }}\right)}{d / s}
$$


where Max $x_{\text {threshold }}$ is the maximum allowed E2E latency (i.e., $250 \mu \mathrm{s}$ ) for the fronthaul link, WE2E $E_{\text {latency }}$ is the worst-case E2E latency experienced by a stream over the fronthaul link that can be computed from the Equation (4), and $d / s$ is the propagation delay.

\section{Description of the Proposed Transport System}

Figure 2 illustrates the proposed EFBNs architecture to transport the multiple eCPRI traffic streams simultaneously between the RRHs and the BBU pool. A traffic stream is defined as a continuous bit rate transmission of the IQ packets at different eCPRI line rates that reserved the MFH bandwidth throughout the communications. However, with the improvement of bandwidth utilization in Ethernet-bridged networks, the E2E latencies for all the RRHs could not be minimized within the eCPRI limits [18]. This is because under high-load conditions and due to the rigorous requirements of the eCPRI, a few RRHs streams would experience the intolerable E2E latencies. Therefore, an optimized path selection algorithm with a low-latency packet forwarding procedure was highly desirable to retain the QoS parameters of EFBNs within the limits, and thus is proposed in this study.

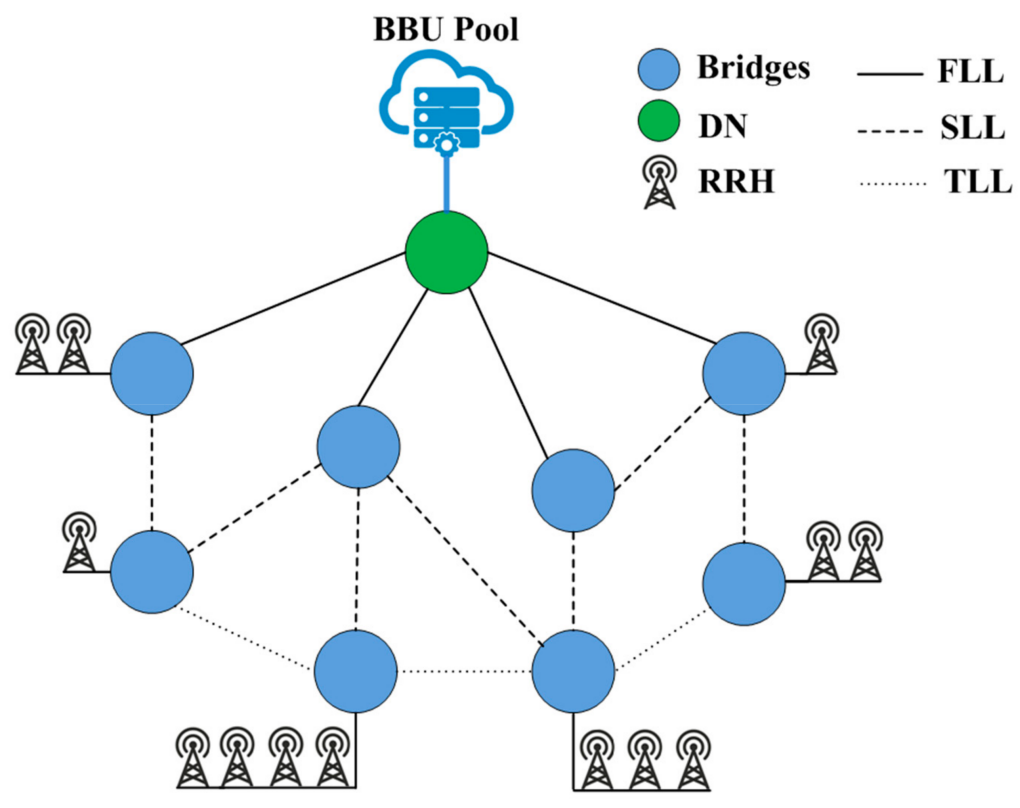

Figure 2. Proposed Ethernet-based fronthaul bridged networks.

\subsection{E2E Latency-Aware Path Computation (LAPC) Procedure}

In the considered fronthaul networks, a software-defined central controller (SDCC) [33] before the transmissions get a global view of the topology by collecting an information metric using the IS-IS (intermediate system to intermediate system) protocol, which was extended by IEEE 802.1Qca [34] for the time-sensitive networking. The IS-IS protocol provided the details about the connection of nodes, link capacities, costs of the links, and distances between the nodes of the topology. Typically, the numbers of RRHs connected to the nodes and accordingly configured eCPRI line rates of the RRHs are well determined in a fronthaul topology [26]. We assumed that all the nodes to which the RRHs are linked also connected with the SDCC, and a path computation element (PCE) is employed at the SDCC. The placement of the BBU pool is also predetermined in the topology, which is preferably connected to a node with which the maximum numbers of nodes are linked as shown in Figure 2. The PCE generates the initial paths for all the eCPRI-based RRHs streams in accordance with the k-th shortest paths algorithm [35] and maintains the paths in a global matrix. The SDCC selects the shortest paths for the individual traffic streams using the SPB algorithm based on the link costs, loads, and delays constraints. Then, for each stream, the SDCC calculates the E2E latency using equations (1) to (4) and updates the latencies in the global matrix along with the pre-selected shortest paths. Alternative paths 
are allocated to the traffic streams that could not satisfy the E2E latency requirements of the eCPRI, such as yield more than $250 \mu$ s. For this purpose, the first traffic load from the over-utilized adjacent links (ALs) of the destination node (DN) are offloaded, and then from the other links of previous ALs and so on. For simplicity, ALs are referred to as the first-level links (FLLs), the links that are adjacent to the FLLs are referred to as the second-level links (SLLs), next after the SLLs are referred to as the third-level links (TLLs), and so on, up to N-level links (NLLs), as illustrated in Figure 2. In this mechanism, the traffic loads from the FLLs to NLLs are systematically offloaded and uniformly distributed on the links. When a traffic load from a high-load link is shifted to a low-load link on each iteration, the according routes of the high-latency experiencing streams that were passing through the over-utilized high-load links are updated in the matrix. These routes were selected by searching from the k-th shortest paths by ensuring that it was not previously selected; then, E2E latencies on the new routes for the streams are recomputed. In the case when offloading the load from the high-load links results in E2E latencies that are greater than the threshold, under such scenarios, those paths are selected for the RRH traffic streams that yield the minimum E2E latencies for the maximum number of traffic streams. The above procedure is repeated until the E2E latencies of all the RRH streams reach the lowest level, and the further shifting of traffic loads between the links does not minimize the latencies. The global matrix contains the routes for each RRH traffic stream along with the E2E latencies computed with the proposed scheme. The output such as the global matrix of the low-latency paths computation algorithm will be used as the input of the low-latency packets transmission scheme, which is proposed in the next section. The proposed low-latency packet forwarding scheme cut down the queuing delays of those RRHs traffic streams, which could not comply with the stringent E2E latency requirements of the eCPRI, despite the low-latency paths computation procedure. The procedure of the proposed low-latency path computation scheme is described in Algorithm 1.

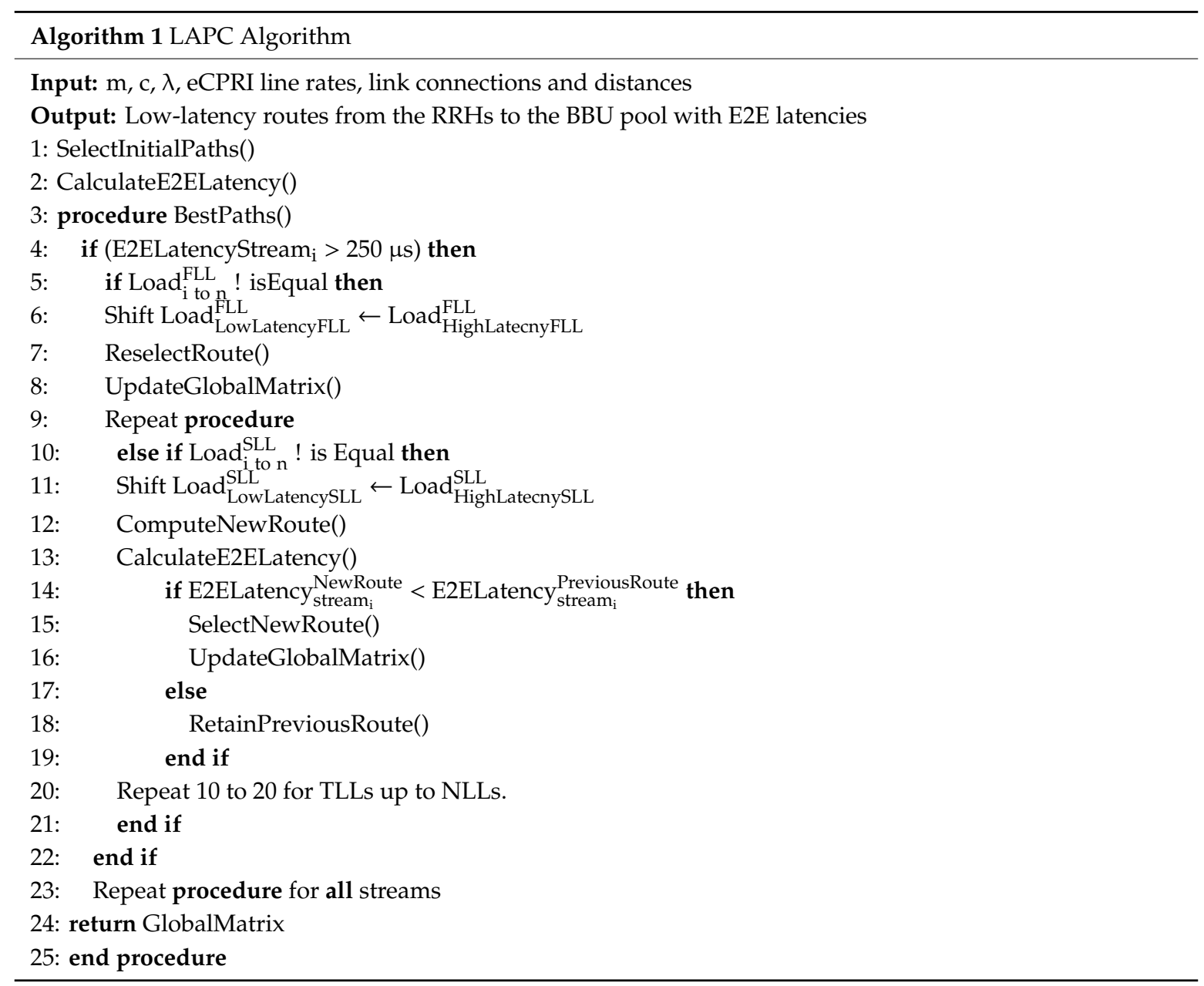




\subsection{Low-Latency Packet Forwarding (LLPF) Scheme}

IEEE P802.1 Qbu [22] introduces a concept of frame preemption (FP) to transport the different priority traffics in time-sensitive Ethernet-bridged networks. In a frame preemption operation, the transmission of low-priority (preemptable) frames is suspended to allow the transmission of one or multiple high-priority (express) frames. After that, the transmission of low-priority frames from the same point is resumed. A low-priority frame that was in the process of transmission has not been completely transmitted, but is rather preempted and buffered until no high-priority frame is left in the queue. The preemptive queuing system enables the cut-through transmission of time-critical packets and minimizes the queuing delays. With this approach, the high-priority frames are transmitted through the switching nodes at low queuing delays without waiting in the queues for the complete transmission of low-priority frames. However, in fronthaul bridged networks, all the eCPRI streams have an equal priority, and simply employing the concepts of frame preemption is unfeasible for traffic streams of equal priority. Therefore, a mechanism that not only allocates the low-load routes to eCPRI traffic streams but also employs the sophisticated priority schemes such as frame preemption to minimize the queuing delays of equal priority eCPRI streams would be more feasible, as discussed below.

Under high traffic load conditions, a few RRH traffic streams are expected to experience the high queuing delays, despite the load balancing and efficiently utilizing the bandwidths [18], especially those that have long routes toward the BBU pool in term of delays and hop counts. As a result, the E2E latencies of such streams become intolerable. Thus, these high-latency experiencing RRHs streams (HERSs) can be given priority over the low-latency experiencing RRHs streams (LERSs) that have a margin of bearing slight queuing delays at intermediate nodes due to their physical or logical locality of being closer to the BBU pool. By considering the packets of HERSs as express frames and packets of LERSs as preemptable frames, the HERSs can be routed at low queuing delays through the nodes that the LERSs are connected to, and eventually, E2E latencies can be minimized. The global matrix of the LAPC algorithm provides the details about the high-latency experiencing RRHs streams over the most optimal paths. However, to manage the impact of preemption and retain the E2E latencies of the LERSs within limits, a frame of LERS should be delayed only a predefined number of times. The simulation result shows that a frame of the LERS can be delayed at maximum four to seven times under the considered scenarios, and after this threshold, a preempted frame of the LERS would be transmitted first instead of an express frame. In the case where a preempted frame is not transmitted according to a predetermined threshold, the E2E latencies of HERS might be low, but it will increase the E2E latencies of the LERS. The preempted frames are reassembled at the next nodes before processing so that these appear as valid Ethernet frames to the physical layer [22]. In the case, when more than one of the HERSs arrive at the bridges of a route, the HERSs that are connected with the most distant bridges, which is in terms of delays and hop counts from the BBU pool, would be given priority over the one with less distant connected bridges. As specified in the standard [22], packet tags are allocated to the preempted frames of the different streams that will be used at the receiver to reassemble the preempted frames. An example of the frames' arrival on the ingress ports, the preemption of frames, and transmissions of frames on the output port of the bridge are depicted in Figure 3. It can be observed that the HER streams of two different priorities (i.e., the priority of stream-3 is more than the priority of stream-2) and a LER stream of low priority (i.e. priority of stream-2 is more than the priority of stream-1) arrived at the ingress ports of the bridge. These priorities can be determined based on the information obtained from the global matrix of the proposed LAPC algorithm. It can be observed in Figure 3 that based on the priorities, the frames of high-priority HERS (i.e., stream-3) preempted the frames of low-priority HERS (i.e., stream-2); then frames of low-priority HERS (i.e., stream-2) preempted the frames of LERS (i.e., stream-1). The new and previously preempted frames of the LERS would be transmitted only when there is no high-priority frame left in the queues or a predefined threshold for the preempted frames is reached. As a result of the above procedure, the high-latency 
experiencing streams that were given more priority in the considered scenarios would experience the low queuing delays, and ultimately, the E2E latencies of all the eCPRI streams can be mitigated.

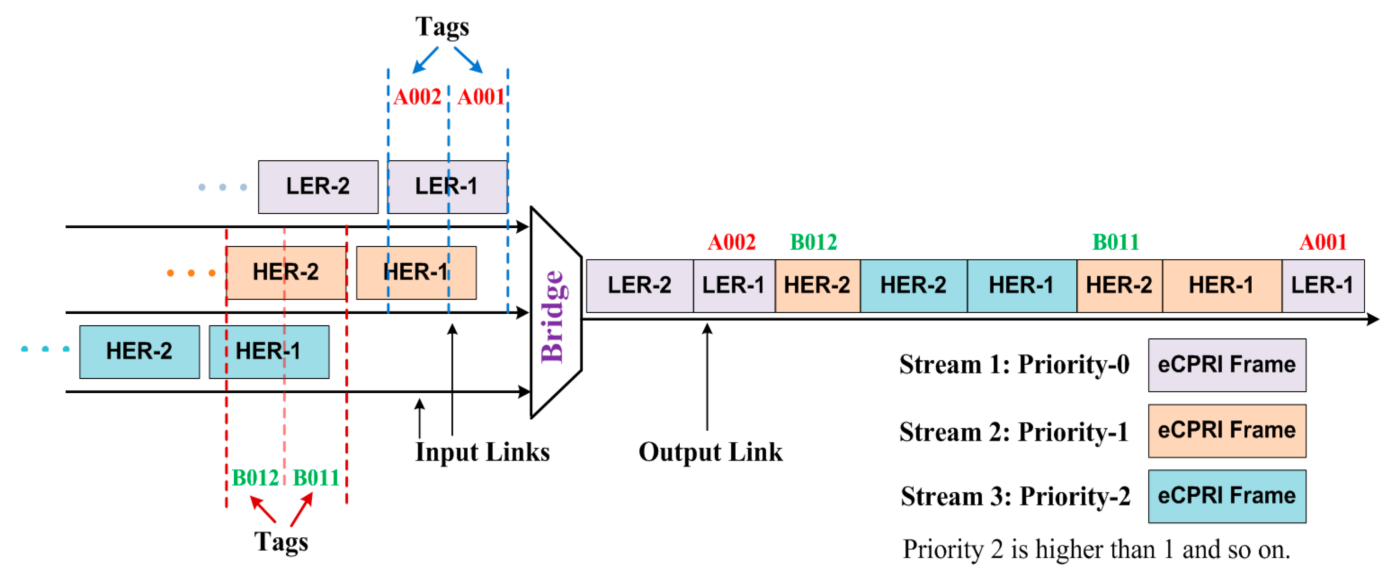

Figure 3. Frame preemption mechanism for enhanced common public radio interface (eCPRI) streams in the Ethernet-based fronthaul bridged networks (EFBNs).

The proposed schemes have certain implementation requirements to be deployed in the considered Ethernet-based fronthaul bridged networks. To compute the low-latency paths in the LAPC scheme, the values of link capacities, link distances, and link costs would be known to the algorithm, which can be determined with the IS-IS protocol. The number of connected RRHs, the configured eCPRI line rates on the RRHs, the size of eCPRI bursts, and the placement of the BBU pool in the topology are well determined in the fronthaul network [18]. The low-latency packet forwarding scheme mainly uses the output values of the LAPC scheme (i.e., global matrix) and requires the bridging nodes that can support the frame preemption operations. Both schemes require that the intermediate bridges should be reconfigurable so that the proposed schemes could be programmed. This functionality can be achieved in the fronthaul networks by using the worldwide available OpenFlow switches with the SDN techniques [19]. The proposed frame preemption-based mechanism is easy to implement and less complex as compared to the legacy packet forwarding schemes such as traffic scheduling [23]. The study [26] reported that due to the utilization of the traffic scheduling techniques in the fronthaul networks, the computational time increment exponentially with the increase of incoming traffic streams at the bridges, which makes it impractical for the multiple bridged networks. In the proposed EFBNs systems, the whole procedure of path selection, E2E latency computation, and accordingly enabling the preemption on the different routes is completed before the transmissions. During the transmissions, the controller periodically collects the topology information using the IS-IS protocol, and the above procedure is executed only when the number of connected RRHs, eCPRI line rates, or link capacities are modified. The proposed scheme yields the computation time up to 1.5 minutes for $100 \mathrm{Gbps}$ of eCPRI traffic load in the considered fronthaul scenarios, which reduces with the reduction of traffic load or the number of connected RRHs in the topology. The path reconfiguration is executed only when the physical topology changes, which typically changes infrequently. Thus, the several minutes of computation time before the transmissions is not a problem or an issue of secondary importance as long as the latency parameters are within the limits, which are the main concern of fronthaul networks $[18,36]$. Moreover, the computational time can be further minimized by parallelizing the calculation for the proposed schemes.

\section{Simulation Setup}

The proposed schemes are evaluated with MATLAB programs. To verify the generality of the schemes, we employed the network topologies COST239 and ARPA2 [37], which are widely used topologies in evaluations of carrier networks [36]. 
IEEE 1914.3 [20] defines the two encapsulation techniques (namely structure-agnostic and structure-aware) to carry the CPRI/eCPRI streams by mapping in the Ethernet frames. In this work, we employed the structure-agnostic mapping scheme to encapsulate the eCPRI packets in the standard Ethernet frames. In this scheme, eCPRI packets are mapped in the Ethernet frames without the knowledge of framing protocols, while in structure-aware encapsulation, eCPRI packets are broken into antenna-carrier and control-data components to transport through the Ethernet switching nodes.

The RRHs connected to the bridging nodes are configured to support the different eCPRI line rates. The RRHs periodically transmit the eCPRI-based bursty traffic toward the BBU pool, which is independent of the end user's data rate and reserves the MFH bandwidth, even when no user is connected with the RRHs. Each RRH is configured to support a single eCPRI line rate randomly from the eCPRI line rate options 1 to 7 such as $0.61 \mathrm{Gbps}, 1.22 \mathrm{Gbps}, 2.45 \mathrm{Gbps}, 3.07 \mathrm{Gbps}, 4.91 \mathrm{Gbps}$, $6.14 \mathrm{Gbps}$, and $9.83 \mathrm{Gbps}$, respectively [10]. In contrast, an eCPRI line rate represents the number of IQ samples that an MFH link can transport between the RRH and the BBU pool [11]. Based on the configured line rates, the RRHs could transmit 50 to $100 \mathrm{Gbps}$ of cumulative eCPRI traffic toward the BBU pool. However, for generality, loads are randomly connected to the bridging nodes. This is because the distribution of RRHs is determined by the demand distribution in reality $[10,18]$. In the considered EFBNs, each RRH sends a burst of 9000 bytes after every 2 ms toward the BBU pool based on [38] for the backward compatibility with the long term evolution-advanced (LTE-A) standards. The maximum size of the Ethernet payload is considered to be 1500 bytes.

The RRHs are connected to bridges through a link of $0.2 \mathrm{~km}$ length at the bandwidth of $10 \mathrm{Gbps}$. It is considered that all the bridges are located within an area of $20 \mathrm{~km}^{2}$. The link distance between the consecutive bridges is randomly determined from 1 to $5 \mathrm{~km}$ similar to realistic networks [18]. The link bandwidth between two bridges is considered to be $10 \mathrm{Gbps}$. In each topology, the BBU pool is connected to a node where a maximum number of links are connected. In the considered scenarios, the BBU pool is connected to the bridging node- 3 and to the bridging node-7 in COST239 and ARPA2 topologies, respectively. The link bandwidth between the BBU-DN is assumed as $100 \mathrm{Gbps}$ and the link length is assumed as $0.2 \mathrm{~km}$. Figure 4 shows the experimental setup to reflect the aforementioned configurations and parameters for simulating the fronthaul scenarios. As shown in Figure $4 a, b$, the RRHs are configured to support the cumulative eCPRI traffic of approximately $50 \mathrm{Gbps}$ and $60 \mathrm{Gbps}$ for COST239 and ARPA2 topologies, respectively. Analogous to this, the cumulative eCPRI traffic loads of 70 to $100 \mathrm{Gbps}$ are formulated during the simulations for both topologies by randomly connecting the RRHs with different nodes at different line rates. The link length between the RRH bridges and DN-BBU pool is considered as $0.2 \mathrm{~km}$. The link lengths between bridges and bridges would be determined randomly from 1 to $5 \mathrm{~km}$. The bandwidth of all the links is considered to be 10 Gbps except for the link between the DN and the BBU pool, which has $100 \mathrm{Gbps}$ of bandwidth.

It is assumed that all the bridges of the fronthaul networks are capable of implementing the frame preemption principles and policies, as discussed in Section 4.2. However, the frame preemption introduces a certain overhead for the preempted frames, which could impact the performance. Therefore, in order to realize the effect of preemption overhead in the EFBNs, the overhead per preemption is estimated as $124 \mathrm{~ns}$, which is equivalent to the processing time of a 155-byte packet [39]. In order to obtain the average of the results, we repeated the simulations 10 times for different topologies and traffic loads. Furthermore, the proposed work has been compared with the LLR scheme [18]. This comparison is reasonable, because the LLR scheme is the most recent development on the given topic, and is considered to be one of the effective solutions to select the low-load paths in fronthaul bridged networks. 


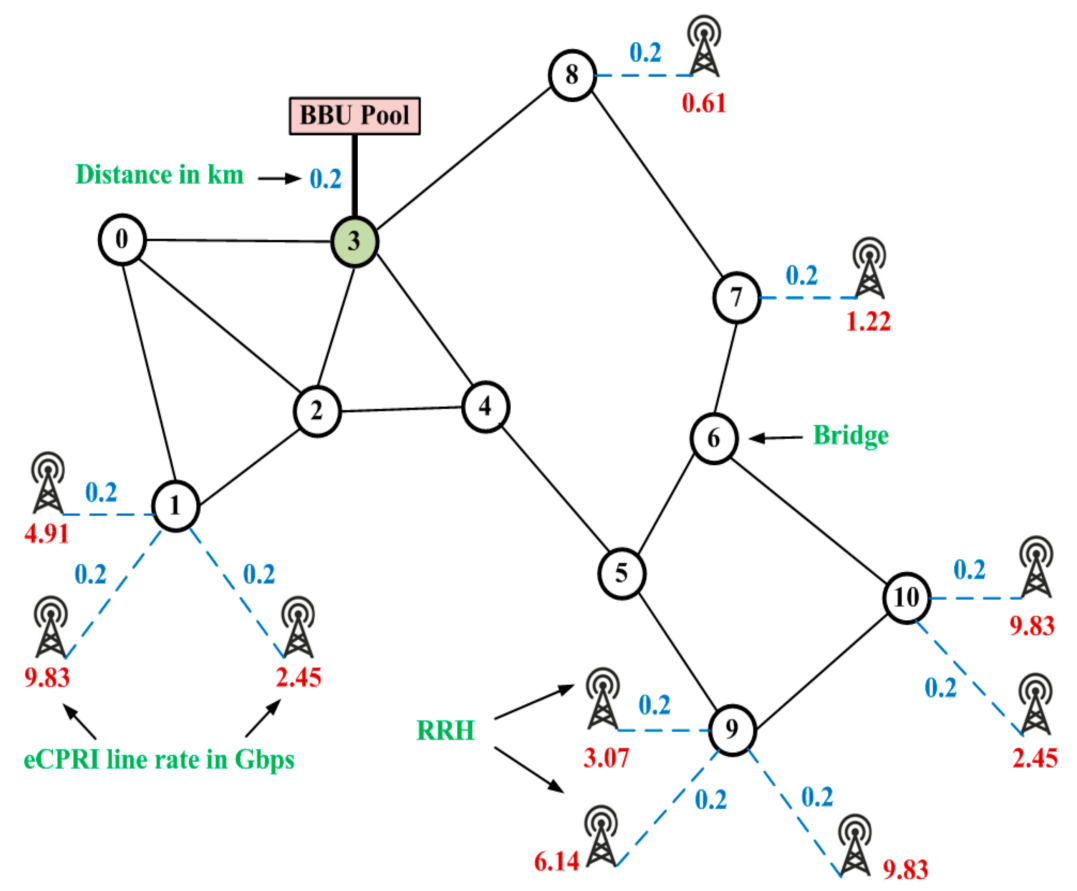

(a)

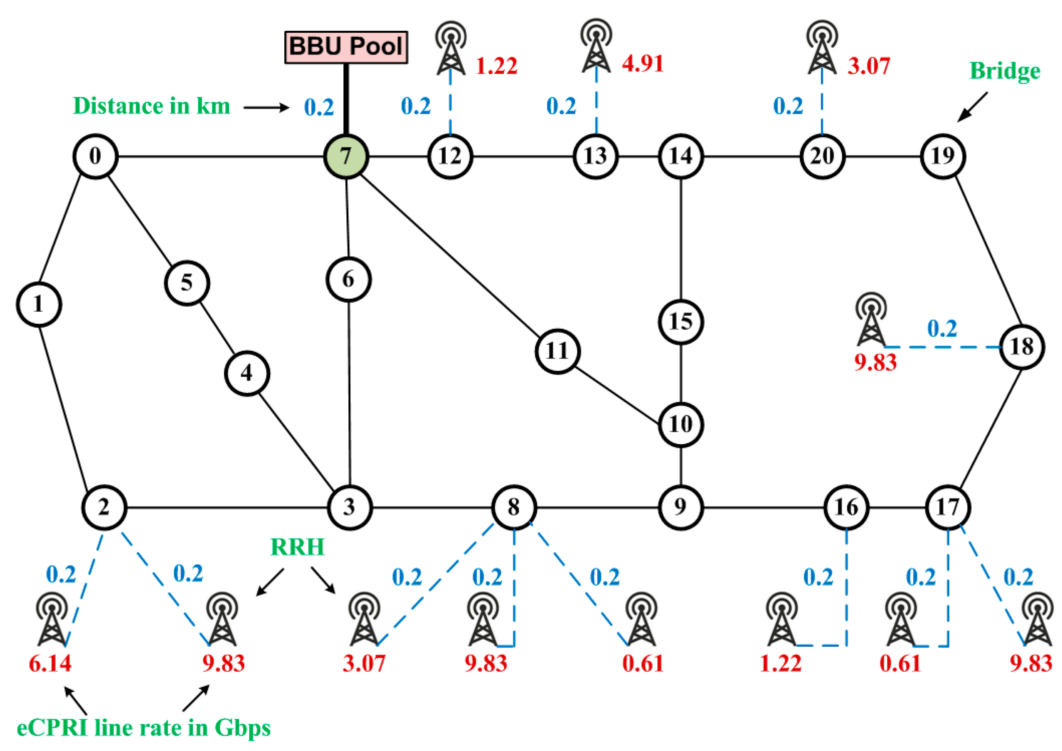

(b)

Figure 4. Considered network topologies for the evaluation of the proposed and existing schemes.

(a) COST239; (b) ARPA2.

\section{Results and Discussion}

Figure 5 a illustrates the average queuing delays experienced by the RRHs eCPRI streams for the COST239 topology while communicating with the BBU pool. The results showed that with the LLR scheme in the EFBNs, the average queuing delays increment as the incoming eCPRI data rates increase. This scheme resulted in the queuing delays up to $300 \mu \mathrm{s}$ under the full load conditions, which are much higher than the required ranges of the fronthaul networks. The reason for the LLR scheme's inefficiency is that this scheme managed the delays by selecting the neighboring paths of the shortest paths, and under the high-load conditions, congestions at the neighboring routes were also high, which resulted in high queuing delays and ultimately increased the latencies to an unacceptable 
level. Our proposed low-latency path computation scheme more efficiently minimized the queuing delays, even under the high traffic load conditions. As shown in Figure $5 \mathrm{a}$, the queuing delays with the LAPC scheme were reduced to $225 \mu$ s for $100 \mathrm{Gbps}$ of eCPRI traffic. Further minimization of the queuing delays was realized by employing the proposed frame preemption concepts along with the low-latency path computation mechanism on the high-load paths. As shown in Figure 5a, the frame preemption-based LLPF scheme, which was named the LLPF-FP, more adroitly alleviated the queuing delays and enabled transmissions of up to $100 \mathrm{Gbps}$ of traffic while retaining delays of less than $200 \mu \mathrm{s}$. With analogy to COST239, similar trends for ARPA2 were shown by the proposed schemes, as depicted by Figure $5 \mathrm{~b}$. The queuing delays for ARPA2 with the LAPC scheme were raised up to $265 \mu \mathrm{s}$ for $100 \mathrm{Gbps}$ of eCPRI traffic, while the LLR resulted beyond the $352 \mu \mathrm{s}$ for the same load. This increment in delays is because of the increased in the propagation delays and the number of the bridges between the RRHs and the BBU in ARPA2 topology. However, the LLPF-FP scheme in ARPA2 topology retained the queuing delays for less than $248 \mu \mathrm{s}$, even when traffic load was high, which verifies the competency of the proposed mechanism to effectually minimize the queuing delays in time-sensitive EFBNs.

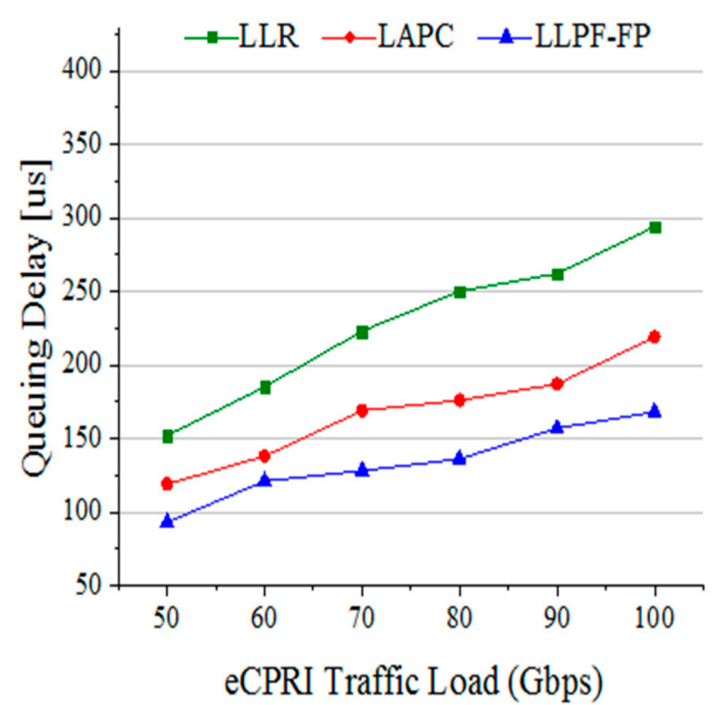

(a)

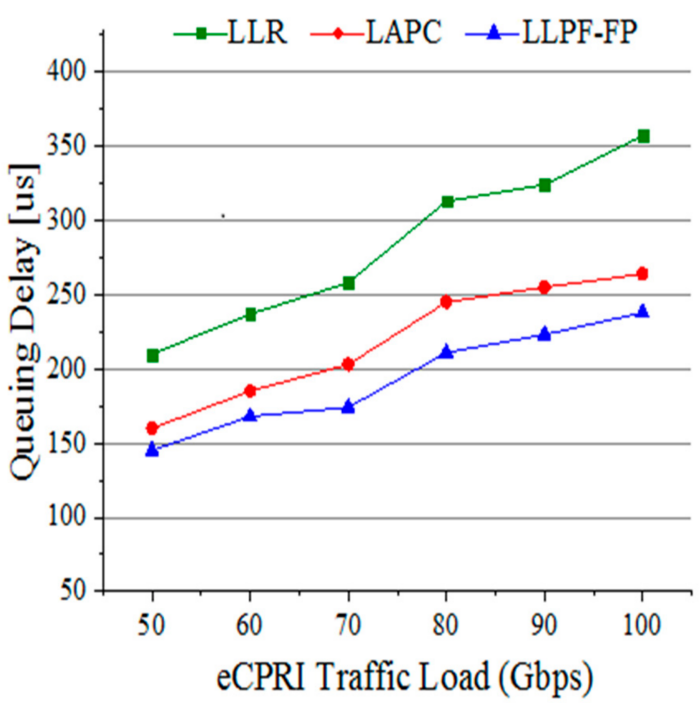

(b)

Figure 5. Performance results for average queuing delays for the (a) COST239; and (b) ARPA2.

Figure 6a depicts the worst-case E2E latencies experienced by the eCPRI streams with the LLR, LAPC, and LLPF-FP schemes. It can be observed that the LLR resulted in high E2E latencies for COST239, which exceeded beyond the $250 \mu$ s limits once the eCPRI traffic load increased to $60 \mathrm{Gbps}$. Whereas, for ARPA2 topology, as shown in Figure 6b, the LLR scheme lacked the mechanism to transport even $50 \mathrm{Gbps}$ of traffic, and resulted in intolerable E2E latencies. Our proposed LAPC algorithm improved the E2E latency performance in the considered EFBNs topologies as compared to existing schemes such as the LLR. The simulation result showed that the LAPC can transport up to $82 \mathrm{Gbps}$ and $65 \mathrm{Gbps}$ of traffic in COST239 and ARPA2 topologies, respectively, by fully satisfying the E2E latency constraints of the fronthaul systems. The LLPF-FP scheme further improved the performance of the EFBNs and enabled the successful transmission of eCPRI traffic up to 100 Gbps for COST239 while retaining the E2E latencies within the threshold. In ARPA2 topology—which has comparatively long routes and more numbers of intermediate bridges with respect to COST239-the proposed LLPF-FP scheme transmitted up to $85 \mathrm{Gbps}$ of traffic without violating the threshold. This verifies the feasibility of our proposed scheme to transport the time-sensitive eCPRI streams in low-cost Ethernet fronthaul networks without deploying the expensive optical fronthaul networks. Under the considered scenario and based on the results for ARPA2 topology, with a successful transmission of $85 \%$ of fronthaul traffic through the low-cost Ethernet infrastructure, a significant amount of CAPEX and OPEX, which 
would be required to deploy the complete optical fronthaul networks, can be saved. Hence, in this case, only $15 \%$ of the traffic required transmission through high-capacity and expensive optical links. For networks such as COST239, almost all of the traffic can be transported through the Ethernet systems, which would be highly cost-effective.

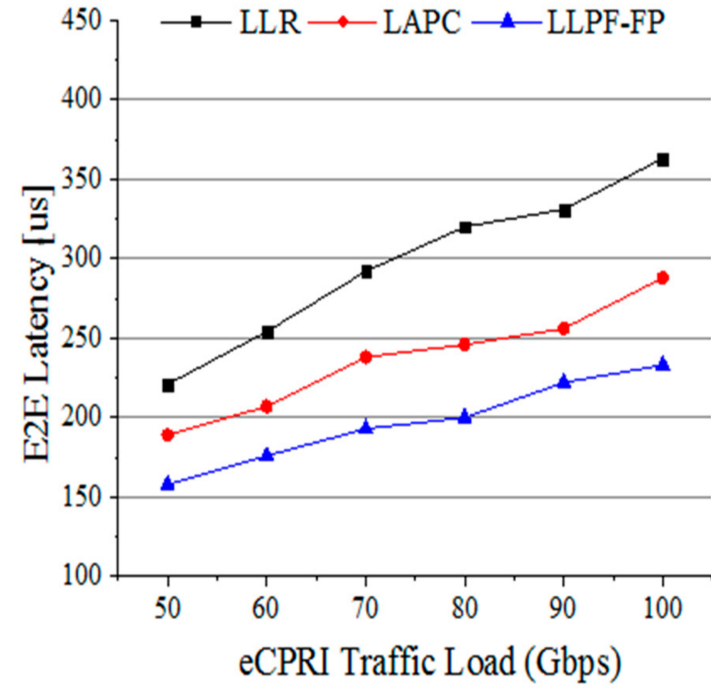

(a)

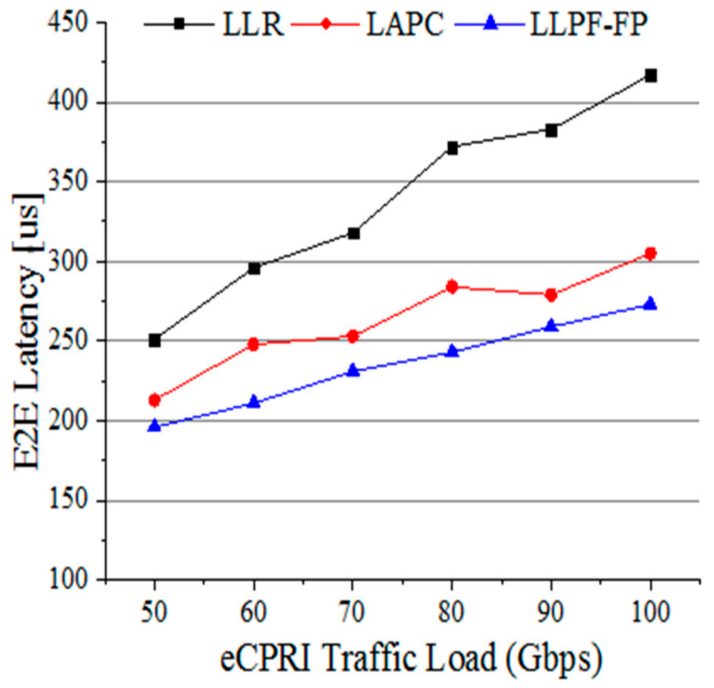

(b)

Figure 6. Performance results for worst-case end-to-end (E2E) latency for (a) COST239; and (b) ARPA2.

The results in Figure 7 show the distances that different schemes can support under different traffic load conditions based on the latency constraints. The distances are computed based on Equations (1)-(5). The proposed LAPC and LAPF-FP schemes outperform the LLR and considerably improve the distances supported by the fronthaul links. It can be observed that with the LAPF-FP scheme, the distances of the fronthaul segment can be increased up to $30 \mathrm{~km}$ for $50 \mathrm{Gbps}$ of traffic, which reduced with the increase of traffic load, as shown in Figure 7a. In ARPA2 topology, as the number of intermediate bridges and distances between the RRHs and BBU pool has been increased as compared to COST239. As a result, the processing, transmission, queuing, and encapsulations delays at each bridging node would also be increased, which resulted in high E2E latencies based on Equation (4), and low fronthaul distances based on Equation (5). As shown in Figure 7b, the proposed LAPF-FP scheme can support fronthaul link distances from $3 \mathrm{~km}$ to $18 \mathrm{~km}$ for $50 \mathrm{Gbps}$ to $100 \mathrm{Gbps}$ of traffic, respectively. In contrast, the existing scheme can only support a maximum link distance of $4.5 \mathrm{~km}$ in Ethernet-based fronthaul bridged networks. The simulation results showed that in about $50 \%$ of cases, the proposed LAPF-FP scheme minimized the queuing delays and worst-case E2E latencies by more than $110 \mu$ s and $90 \mu$ s, respectively, compared to the LLR. In about $70 \%$ of cases, our scheme improved the link distances up to $35 \%$ compared to the LLR.

The results show that alone, delay-sensitive path computation schemes could not be enough to maximize the traffic transmissions in the EFBNs, and along with the path computation schemes, novel traffic forwarding concepts such as the one proposed in this study that enabled the frame preemption on high-load paths were required to fully exploit the advantages of leveraging Ethernet-based fronthaul networks. The simulation results showed a tradeoff between the maximum link distances and acceptable E2E latencies. By improving the E2E latencies in fronthaul networks, the length of Ethernet links can be increased. It can be concluded that with frame preemption in the proposed low-latency packet forwarding which is named the LAPF-FP scheme, the E2E latencies of maximum eCPRI streams can be retained closer to the threshold, and long-distance Ethernet links can be deployed between the RRHs and the BBU pool by fully satisfying the latency constraints of the fronthaul networks. However, 
this type of performance is not guaranteed with the existing schemes, which makes them less suitable to addressing the latency constraints of Ethernet-based fronthaul bridged networks.

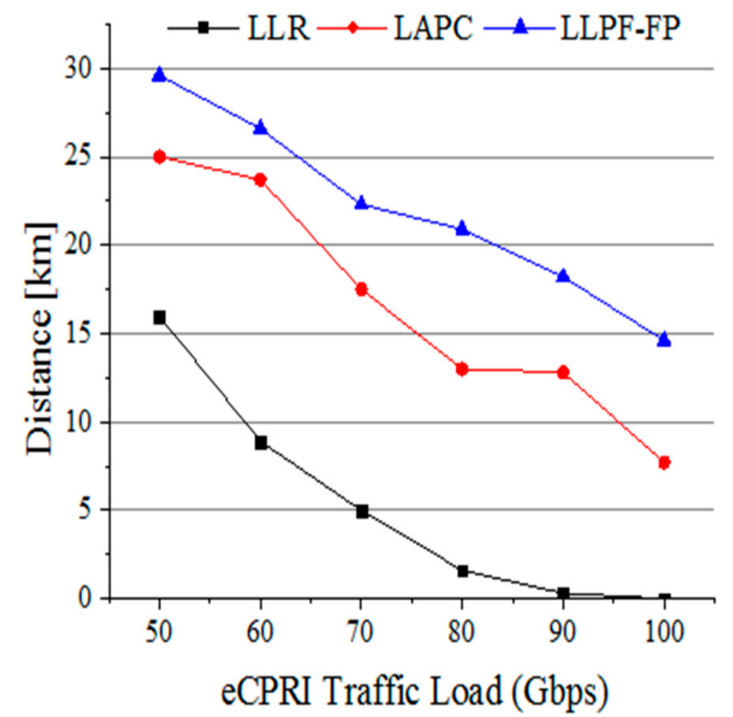

(a)

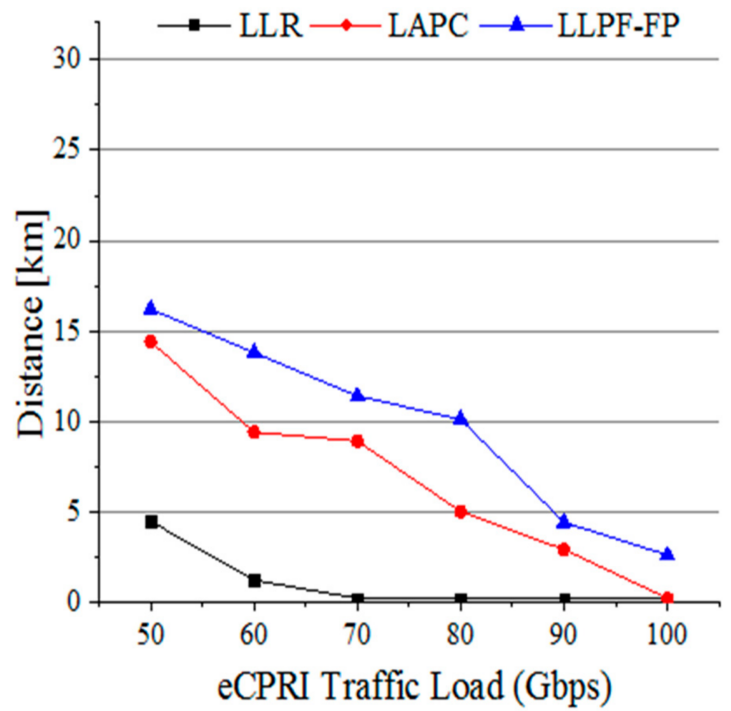

(b)

Figure 7. Fronthaul distances supported by the different schemes for the (a) COST239; and (b) ARPA2.

\section{Conclusions}

We proposed the end-to-end (E2E) latency-aware path computation (LAPC) scheme to improve the link utilization in capacity constraint Ethernet-based fronthaul bridged networks (EFBNs). The path computations were performed by primarily focusing on the E2E latency constraints of the eCPRI traffic streams. The latency-aware path selection scheme assures the lowest E2E latencies in the fronthaul bridged networks by considering the frame level queuing delays of eCPRI bursts. The performance of the EFBNs was further improved by exploiting the concepts of frame preemption in the proposed low-latency packet forwarding scheme, which is named the LAPF-FP. The LAPF-FP scheme reduces the queuing delays of high-latency experiencing RRHs (remote radio heads) streams by slightly increasing the queuing delays of the low-latency experiencing RRHs streams without the degradation of the overall system performance. The simulation results confirmed that the LAPF-FP scheme considerably reduced the E2E latencies and intensified the low-cost Ethernet systems to transport the time-sensitive eCPRI traffic up to $100 \mathrm{Gbps}$ at tolerable latencies. Moreover, the low E2E latencies due to the LAPF-FP scheme significantly improved the link distances between the RRHs and the baseband unit (BBU) pool. This led to an expectation that the leveraging Ethernet systems could be a promising solution to implement the fronthaul bridged networks in the future cloud radio access networks (C-RANs). In summary, the following are the key findings from this study:

1. This study considered the queuing delays and end-to-end latencies for computing and selecting the optimal paths for individual eCPRI traffic streams in the EFBNs.

2. A novel packet forwarding mechanism for equal priority eCPRI streams has been proposed that maximizes the transmissions of multiple eCPRI streams at tolerable latencies as well as improves the link distances between the RRHs and the BBU pool.

3. The simulation results showed that in about $50 \%$ of cases, the proposed scheme minimized the queuing delays and worst-case E2E latencies by more than $110 \mu \mathrm{s}$ and $90 \mu \mathrm{s}$ compared to the LLR. In about $70 \%$ of cases, our scheme improved the link distances up to $35 \%$ as compared to the LLR. This level of performance is not guaranteed with the existing schemes. 
4. With the proposed schemes, time-sensitive eCPRI streams can be transported through the leveraging Ethernet links, which significantly reduced the CAPEX and OPEX of deploying extortionate optical-based fronthaul bridged networks in envisioned 5G cloud-RANs.

Author Contributions: Conceptualization, M.W., and A.K.; methodology, M.W.; software, M.W.; validation, M.W., and A.K.; formal analysis, A.K.; investigation, M.W.; resources, A.K.; data curation, M.W.; writing-original draft preparation, M.W.; writing-review and editing, A.K.; visualization, A.K.; supervision, A.K.; project administration, A.K.; funding acquisition, A.K.

Funding: This work was supported in part by the National Research Foundation (NRF) and Ministry of Science, ICT and Future Planning (MSIP) of South Korea under grant number 2018R1D1A1B07049877.

Conflicts of Interest: The authors declare no conflict of interest.

\section{References}

1. Cisco Visual Networking Index, Global Mobile Data Traffic Forecast Update, 2017-2022, Cisco White Paper. 2019. Available online: https://www.cisco.com/c/en/us/solutions/collateral/service-provider/visualnetworking-index-vni/vni-forecast-qa.pdf (accessed on 9 June 2019).

2. Luong, P.; Gagnon, F.; Despins, C.; Tran, L. Joint virtual computing and radio resource allocation in limited fronthaul green C-RANs. IEEE Trans. Wirel. Commun. 2018, 17, 2602-2617. [CrossRef]

3. Musumeci, F.; Silva, E.; Tornatore, M. Enhancing RAN throughput by optimized CoMP controller placement in optical metro networks. IEEE J. Select. Areas Commun. 2018, 36, 2561-2569. [CrossRef]

4. Das, S.; Ruffini, M. A variable rate fronthaul scheme for cloud radio access networks (C-RAN). J. Lightwave Technol. 2019, 37, 3153-3165. [CrossRef]

5. 3rd Generation Partnership Project (3GPP) Working Group, Tech. rep. 38.801. Study on New Radio Access Technology-Radio Access Architecture and Interfaces. Version 14.0.0. 2017. Available online: https://www.3gpp.org/ftp/Specs/archive/38_series/38.801/38801-e00.zip (accessed on 9 June 2019).

6. Zhou, Y.; Li, J.; Shi, Y.; Wong, V.W.S. Flexible functional split design for downlink C-RAN with capacity-constrained fronthaul. IEEE Trans. Vehic. Technol. 2019. [CrossRef]

7. Frascolla, V.; Dominicini, C.; Paiva, M.; Caporossi, G.; Marotta, M.; Ribeiro, M.; Segatto, M.; Martinello, M.; Monteiro, M.; Both, C. Optimizing C-RAN backhaul topologies: A resilience-oriented approach using graph invariants. App. Sci. 2019, 9, 136. [CrossRef]

8. Common Public Radio Interface (CPRI), Interface Specification 2015, Version 7.0. Available online: http: //www.cpri.info/downloads/CPRI_v_7_0_2015-10-09.pdf (accessed on 9 June 2019).

9. Molner, N.; Oliva, A.; Stavrakakis, I.; Azcorra, A. Optimization of an integrated fronthaul/backhaul network under path and delay constraints. Ad Hoc Nets. 2019, 83, 41-54. [CrossRef]

10. Common Public Radio Interface (CPRI), eCPRI Interface Specification 2019, Version 2.0. Available online: http://www.cpri.info/downloads/eCPRI_v_2.0_2019_05_10c.pdf (accessed on 9 June 2019).

11. Cai, M.; Liu, Q.; Jiang, H. A novel efficient wireless fronthaul (EWF) method for the common public radio interface (CPRI) signal transmission. In Proceedings of the 2018 IEEE 88th Vehicular Technology Conference (VTC-Fall), Chicago, IL, USA, 27-30 August 2018.

12. Nakayama, Y.; Hisano, D.; Kubo, T.; Fukada, Y.; Terada, J.; Otaka, A. TDD-based rapid fault detection and recovery for fronthaul bridged network. IEEE Commun. Lett. 2018, 22, 498-501. [CrossRef]

13. Gomes, N.J.; Sehier, P.; Thomas, H.; Chanclou, P.; Li, B.; Munch, D.; Assimakopoulos, P.; Dixit, S.; Jungnickel, V. Boosting $5 \mathrm{G}$ through Ethernet: How evolved fronthaul can take next-generation mobile to the next level. IEEE Vehic. Technol. Magaz. 2018, 13, 74-84. [CrossRef]

14. Peng, M.; Wang, Y.; Dang, T.; Yan, Z. Cost-efficient resource allocation in cloud radio access networks with heterogeneous fronthaul expenditures. IEEE Trans. Wirel. Commun. 2017, 16, 4626-4638. [CrossRef]

15. Kuipers, F.; Van Mieghem, P.; Korkmaz, T.; Krunz, M. An overview of constraint-based path selection algorithms for QoS routing. IEEE Commun. Magaz. 2002, 40, 50-55. [CrossRef]

16. Waqar, M.; Kim, A.; Yoon, J. A performance study of PON-Based 5G cloud-RANs with frame preemption and strict priority. In Proceedings of the International Conference on Green and Human Information Technology (ICGHIT), Kuala Lumpur, Malaysia, 16-18 January 2019. 
17. Mountaser, G.; Mahmoodi, T.; Simeone, O. Reliable and low-latency fronthaul for tactile internet applications. IEEE J. Select. Areas Commun. 2018, 36, 2455-2463. [CrossRef]

18. Nakayama, Y.; Hisano, D.; Kubo, T.; Fukada, Y.; Terada, J.; Otaka, A. Low-latency routing scheme for a fronthaul bridged network. J. Opt. Commun. Net. 2018, 10, 14-23. [CrossRef]

19. Lin, F.P.; Tsai, Z. Hierarchical edge-cloud SDN controller system with optimal adaptive resource allocation for load-balancing. IEEE Syst. J. 2019, 9, 111-121. [CrossRef]

20. IEEE P1914.3, Draft Standard for Radio Over Ethernet Encapsulations and Mappings. Version 2.0. 2018. Available online: http://sites.ieee.org/sagroups-1914/files/2018/02/tf3_1914.3_D3_0_MEC_clean.pdf (accessed on 9 June 2019).

21. IEEE P802.1CM, Draft Standard for Local and Metropolitan Area Networks-Amendment: Time-Sensitive Networking for Fronthaul. Version 2.2. 2018. Available online: http://www.ieee802.org/1/files/private/cmdrafts/d2/802-1CM-d2-2.pdf (accessed on 9 June 2019).

22. IEEE P802.1Qbu, Draft Standard for Local and Metropolitan Area Networks-Bridges and Bridged Networks-Amendment: Enhancements for Frame Preemption. Version 3.0. 2015. Available online: http://www.ieee802.org/1/files/private/bu-drafts/d3/802-1Qbu-d3-0.pdf (accessed on 9 June 2019).

23. IEEE P802.1Qbv, Draft Standard for Local and Metropolitan Area Networks—Bridges and Bridged Networks-Amendment: Enhancements for Scheduled Traffic. Version 3.1. 2015. Available online: http://www.ieee802.org/1/files/private/bv-drafts/d3/802-1Qbv-d3-1.pdf (accessed on 9 June 2019).

24. Smith, P.A. CPRI Fronthaul Requirements Discussion with TSN. Tech. rep., San Diego, Huawei. 2015. Available online: http://www.ieee802.org/1/files/public/.../new-ashwood-tsn-cpri-fronthaul-0714-v03.pdf (accessed on 9 June 2019).

25. Waqar, M.; Kim, A.; Yoon, J.J. A performance analysis of 5G fronthaul networks for long-distance communications. In Proceedings of the 2019 Wireless Days (WD) Conference, Manchester, UK, 24-26 April 2019.

26. Chitimalla, D.; Kondepu, K.; Valcarenghi, L.; Tornatore, M.; Mukherjee, B. 5G fronthaul-latency and jitter studies of CPRI over Ethernet. IEEE/OSA J. Opt. Commun. Net. 2017, 9, 72-182. [CrossRef]

27. Waqar, M.; Kim, A.; Peter, K.C. A transport scheme for reducing delays and jitter in Ethernet-based 5G fronthaul networks. IEEE Access. 2018, 6, 446110-446121. [CrossRef]

28. Yahya, W.; Basuki, A.; Jiang, J.R. The extended Dijkstra's-based load balancing for OpenFlow network. Int. J. Electr. Comp. Eng. 2015, 5, 289-296.

29. Gurumoorthy, K.B.; Kumar, N. Mutual constraint-based GA suggested routing algorithm for improving QoS in clustered MANETS. Wirel. Person. Commun. 2018, 98, 2975-2991. [CrossRef]

30. Huo, C.; Yuan, J.; Song, G.; Shi, Z. Node reliability based multi-path routing algorithm of high-speed power line communication network. In Proceedings of the 2019 IEEE 4th International Conference on Cloud Computing and Big Data Analysis (ICCCBDA), Chengdu, China, 12-15 April 2019.

31. Guirguis, A.; Digham, F.; Seddik, K.G.; Ibrahim, M.; Harras, K.A.; Youssef, M. Primary user-aware optimal discovery routing for cognitive radio networks. IEEE Trans. Mob. Comput. 2019, 18, 193-206. [CrossRef]

32. IEEE 802.1aq, Draft Standard for Local and Metropolitan Area Networks-Bridges and Bridged Networks —Amendment: Shortest Path Bridging. Version 4.6. 2012. Available online: http://www.ieee802.org/1/files/ private/aq-drafts/d4/802-1aq-D4-6.pdf (accessed on 9 June 2019).

33. Gutierrez, M.; Ademaj, A.; Steiner, W.; Dobrin, R.; Punnekkat, S. Self-configuration of IEEE 802.1 TSN networks. In Proceedings of the 2017 22nd IEEE International Conference on Emerging Technologies and Factory Automation (ETFA), Limassol, Cyprus, 12-15 September 2017.

34. IEEE 802.1Qca, Draft Standard for Local and Metropolitan Area Networks-Bridges and Bridged Networks-Amendment: IS-IS Path Control and Reservation. Version 2.1. 2015. Available online: http://www.ieee802.org/1/files/private/ca-drafts/d2/802-1Qca-d2-1.pdf (accessed on 9 June 2019).

35. Hershberger, J.; Maxel, M.; Suri, S. Finding the k shortest simple paths: A new algorithm and its implementation. ACM Trans. Algos. 2007, 3, 45. [CrossRef]

36. Hisano, D.; Nakayama, Y.; Kubo, T.; Uzawa, H.; Fukada, Y.; Terada, J. Decoupling of uplink user and HARQ response signals to relax the latency requirement for bridged fronthaul networks. IEEE/OSA J. Opt. Commun. Net. 2019, 11, B26-B36. [CrossRef]

37. Lee, D.; Lee, K.; Yoo, S. Efficient Ethernet ring mesh network design. J. Lightweight Technol. 2011, 29, 2677-2683. [CrossRef] 
38. 3rd Generation Partnership Project (3GPP) Working Group, Tech. Rep. 36.213. Evolved Universal Terrestrial Radio Access (E-UTRA); Physical Layer Procedures. Version 14.6.0. 2018. Available online: https://www.etsi.org/deliver/etsi_ts/136200_136299/136213/14.06.00_60/ts_136213v140600p.pdf (accessed on 9 June 2019).

39. Park, T.; Samii, S.; Shin, K.G. Design optimization of frame preemption in real-time switched Ethernet. In Proceedings of the 2019 Design, Automation \& Test in Europe Conference \& Exhibition (DATE), Florence, Italy, 25-29 March 2019.

(C) 2019 by the authors. Licensee MDPI, Basel, Switzerland. This article is an open access article distributed under the terms and conditions of the Creative Commons Attribution (CC BY) license (http://creativecommons.org/licenses/by/4.0/). 